\title{
A Clustering Algorithm for Wireless Sensor Network
}

\author{
Yongxin Feng, Wenbo Zhang* \\ School of Information Science \& Engineering Shenyang Ligong University, \\ Shenyang 110159, China \\ *Corresponding author's E-mail: zhangwenbo@yeah.net
}

\begin{abstract}
In wireless sensor network the bad distribution for cluster head was not taken into account in traditional clustering algorithm such as LEACH algorithm. However the distribution uniformity for the cluster head in wireless sensor networks is very critical, for the better cluster head distribution could effectively save the communication cost and prolong the the lifespan of the network. The math programming approach that we use in this paper is based on a variation of a maximal expected covering location model due to Daskin. Daskin introduced a variant of the MCLP that considers the possibility that facilities may be unable to respond to demand at all times. The resultant model was labeled as MEXCLP (Maximum Expected Covering Location Problem). So we put forward the MEXCLP algorithm for clustering according to the greatest expectations of coverage principle, in this algorithm the cluster head was taken regarded as the provider of services, the cluster head could provide service to the member nodes in the cluster. The algorithm considers the process of service failure (link failure, and so on), and it is based on the assumption of probability of the services failure, a reasonable choice through the head cluster node enables network nodes be served with the largest request number. From the simulation results can be see that the MEXCLP algorithm is more uniformed in finding head cluster, and under the same failure rate circumstances, MEXCLP algorithm may provide more efficient service and consume less energy than the LEACH algorithm. So this clustering algorithm could effectively and efficiently be suitable to the wireless sensor network and provide more reliable service for the sensor nodes in the cluster.
\end{abstract}

Keywords: wireless sensor network; clustering; MEXCLP algorithm; service failure ratio

\section{Introduction}

Recent advancements in integrated circuits have fostered the emergence of a new generation of tiny, inexpensive low power sensors. A sensor network is a set of nodes in which a battery, a sensoring and a wireless communication device are embedded. Sensor networks are a special case of ad-hoc networks with objects generally densely deployed either very close or inside a studied phenomenon. Sensor nodes are deployed over hostile or remote environments to monitor a target area. Therefore, their unreplacable batteries imply energy to be the most important system resource. These objects are expected to work and col- laborate as long as possible in order to send their collected data to one or more sink stations. These sinks, also called monitoring stations, are considered to have unlimited battery and aim to collect information from sensor nodes in multi-hop manner. The lifetime of the network is the time during which the surface coverage is maintained. A point of the target surface is said to be covered if it is in the sensoring range of an active sensor which can report to a sink. It means that the sensor network can accomplish its surveillance task while the set of connected components contain monitoring stations covers the target area. To extend the network lifespan, some nodes are placed into sleeping mode to save their energy. The issue consists in these 
nodes deciding themselves whether to turn off or not so that the whole area remains to be covered and the subset of active nodes could be connected.

Due to their economic and computational feasibility, a network of hundreds and thousands of sensors has the potential for numerous applications in both military and civil applications such as combat field surveillance, security and disaster management. These sensing devices are capable to monitor a wide variety of ambient conditions such as: temperature, pressure, motion etc. The sheer number of these devices and their ad-hoc deployment in the area of interest brings numerous challenges in networking and management of these systems. Sensors are typically disposable and expected to last until their energy drains. Therefore, energy is a very scarce resource for such sensor systems and has to be managed wisely in order to extend the life of the sensors for the duration of a particular mission.

Wireless sensor network clustering algorithm is recognized as a valid method of self-organization. Currently, many protocols applications in the wireless sensor network are depended on the logic network architecture of sub-clusters, so an important research content of wireless sensor network is putting the wireless sensor networks into clustering with a reasonable method.

LEACH algorithm is very typical for the clustering algorithm, which makes nodes in the network cluster according to a certain rule, and selects the head of the cluster to take on the role of data control center, makes data in cluster integrate at home then forward to the SINK nodes, thus reducing the amount of data transmission and the number of transponders, the network will reduce energy consumption. But the election of cluster head is random and can not guarantee the rationality, and it requires cluster head node for longdistance transmission and makes the data sent directly to SINK, which in this large-scale networks is not easily achieved. Although HEED algorithm is different from LEACH algorithm on the selection criteria of cluster head and the mechanism of competition of the cluster head, clustering speed has a certain improvement, taking into account the cluster of communications expenses after cluster, the remaining energy of the nodes as a parameter introduce to the algorithms, making the election cluster better suited to play the task of transmitting data, making the network topology more reasonable, so that the entire network of power consumption will be more evenly, but the algorithm did not consider wireless sensor networks in the link failure or services failure, the cluster head node can not provide services to the node within the cluster . So designing an algorithm, which not only considers the cluster head uniform distribution and balances link but also provides the best service at the circumstance of services failure, is very valuable.

The remainder of this paper is organized as follows. We briefly review related work in Section 2 . The MEXCLP model and cluster formation are presented in details in Section 3, and in this section we present the clustering algorithm. Our simulation result is presented in section 4. Finally, in section 5, we conclude the paper and highlights future directions for other aspects of improvement in WSN.

\section{Related work}

One of the most critical issues in designing sensor network algorithms is to minimize the energy consumption while meeting certain performance requirements such as delay and throughput, etc. Many researchers have focused on issues like energy aware routing, energy saving through activation of a limited subset of nodes, and proposed protocols and algorithms including energy efficiency.

Clustering in wireless sensor network is a hot topic. A cluster-based routing protocol groups sensor nodes in order to efficiently relay the sensed data to the sink. Each group of sensors has a cluster head that is a specified node being less energy-constrained. Cluster heads aggregate the received data and send them to the sink. Cluster forming is a method that minimizes energy consumption and communication latency. Three most well known hierarchical routing protocols are LEACH, TEEN and Chain-Based 3 level PEGASIS. However, most proposed approaches have too many assumptions on sensor nodes. For example, node must have the same initial energy level, nodes are static, or nodes should have much information about other nodes. These assumptions are not practical in reality. Other problems such as in LEACH the cluster head is selected based on a round-robin strategy. Every time, the cluster head changing produces a large overhead since all the nodes in this cluster has to be notified.

Since that when there is an energy difference to some threshold between these nodes in the network, the sensors die out faster than a more uniformed energy-setting [12]. In real life situation, it is difficult for the sensors to maintain their energy uniformly, this makes energy imbalance between nodes to occur easily. LEACH assumes that the energy usage of each node with respect to the overall energy of the system or network is 
homogeneous. Conventional protocols such as Minimum Transmission Energy (MTE) and Direct Transmission (DT) [11] do not also assure a balanced and uniformly use of the sensor's respective energy as the network evolves. In Distributed Energy Efficient Clustering algorithm (DEEC) [10], a probability based clustering algorithm was proposed. DEEC elects cluster heads based on the knowledge of the ratio between residual energy of each nodes and the average energy of the network. This knowledge however requires additional energy consumption to share the information among the sensor nodes. Stable Election Protocol (SEP) [12] is another heterogeneity-aware protocol. It does not require energy knowledge sharing but is based on assigning weighted election probabilities of each node to be elected cluster head according to their respective energy. This approach ensures that the cluster head election is randomly selected and distributed based on the fraction of energy of each ode therefore assuring a uniform use of the nodes energy.

In SEP, two types of nodes (two tier in-clustering) and two level hierarchies were considered. SEP is based on weighted election probabilities of each node to become cluster head according to the remaining energy in each ode. A survey of clustering algorithm was presented in [13]; the even distribution of sensors in clusters is another primary objective of clustering called load balancing that needs to be considered when designing a robust protocol for WSNs. The clustering issue was also discussed in a review in wireless multimedia sensor networks. The contribution of this work is a SEP extension called SEP-E, by considering three-tier node classification in a two-level hierarchical network. The new node type for the purpose of this study is referred to as "intermediate nodes", which serves as a bridge between the advanced nodes and the normal nodes. The intermediate nodes can take on the role of information fusion and filtering depending on the application settings, which we intend to study further. Our goal is to achieve a robust selfconfigured WSN that maximizes its lifetime.

\section{Clustering algorithm based on MEXCLP}

The Capacitated Dynamic MEXCLP is a strategic model. The model assumes that the cluster heads are completely reliable while all links have (identical) steadystate probability of failure. Sensors are mobile with known velocity vectors. The time horizon is divided into equal time periods and relocation of cluster heads which takes place at the beginning of each time period. Cluster heads are identical in all respects. The data transmission is assumed to be negligible and is not transmitted in packets.

In order to incorporate the capacity constraint, each sensor needs to be assigned to one of the cluster heads "covering" it. However, we still have link failure probabilities and correspondingly, need multiple coverage on sensors. Thus the trade off is between multiple coverage and preferential assignment on one side and relocation cost on the other. We present two different formulations based on the way in which capacity constraints are applied. In the first formulation, capacity constraint is applied for each time period while in the second, it is applied for the entire time horizon. Thus the second one can be viewed as a relaxed version of the first.

If the total number of wireless sensor network nodes is $N$, including service nodes $M(M<N), h_{k}$ is the request number of $k$ nodes. Then, the following definition made:

$$
\begin{gathered}
y_{k}= \begin{cases}1 & \text { if the nodes are covered } \\
0 & \text { others }\end{cases} \\
x_{i}= \begin{cases}1 & \text { if the nodes of }{ }^{\prime} i^{\prime} \text { is cluster head } \\
0 & \text { others }\end{cases} \\
a_{i}= \begin{cases}1 & \text { ' } k^{\prime} \text { can be covered by cluster head }{ }^{\prime} i \\
0 & \text { others }\end{cases}
\end{gathered}
$$

Furthermore, these variables have to meet in the following conditions:

$$
\begin{aligned}
& y_{k}-\sum_{k} a_{k} X_{k} \leq 0 \quad k=1, \ldots, N \\
& \sum_{k} X_{k} \leq M \\
& x_{i}=0,1 i=1, \ldots, n \\
& y_{k}=0,1 \quad k=1, \ldots, n
\end{aligned}
$$

It needs to be emphasized that the ' $k$ ' node can be covered depended on the ' $i$ ' node exist facilities and the distance between the ' $k$ ' node and the facilities of ' $i$ ' is less than the radius, the ' $k$ ' node can be covered by the ' $i$ ' node' facilities means that this node can provide services for the ' $k$ ' node, in case of the total number of nodes, the total number of service node and the total request number of each node are known. The first cluster formation process is how to select $\mathrm{M}$ nodes service nodes within the I nodes and make all the nodes can be serviced and the request of the number of service is the Most. That is:

$$
\text { Maximize } \sum_{k} h_{k} y_{k}
$$


At this point, ' $M$ ' service node is the first cluster node, its location is the optimal location of the first cluster. However, wireless sensor networks uses wireless communication mode to communicate, the breakdown of communication link layer and the failure of service happens between nodes, service nodes can not provide services for the nodes within the scope of its services. If the failure probability of services is $p(0$ p 1), and order the probability of failure is equal and not related for all the nodes of its services. When the ' $k$ ' node was serviced by the ' $M$ ' node service, the ' $k$ ' node was serviced successful probability is $1-\mathrm{pm}$, Furthermore, When $M$ service nodes provide services for ' $k$ ' node, the request of the ' $k$ ' nodes were covered by $H_{k, m}$. Then

$$
H_{k, m}= \begin{cases}h_{k} & \text { Service success } \\ 0 & \text { Service failure }\end{cases}
$$

Calculation can induce expectation of $H_{k, m}$ :

$$
\begin{array}{r}
E\left(H_{k, m}\right)=h_{k}\left(1-p^{m}\right) \quad \forall k, m \\
\triangle E\left(H_{k, m}\right)=E\left(H_{k, m}\right)-E\left(H_{k, m-1}\right) \\
=h_{k} p^{m-1}(1-p) \\
m=1,2, \ldots, M
\end{array}
$$

Further more, when the number of cover ' $k$ ' node facilities increase from ' $M-1$ ' to ' $M$ ', it can lead to ' $k$ ' nodes expect increment of requests for coverage is:

$$
\begin{array}{r}
\triangle E\left(H_{k, m}\right)=E\left(H_{k, m}\right)-E\left(H_{k, m-1}\right) \\
=h_{k} p^{m-1}(1-p) \\
m=1,2, \ldots, M
\end{array}
$$

Combination of (1) and (3) can induce

$$
\operatorname{Maximize} \sum_{k=1}^{N} \sum_{j=1}^{M}(1-p) p^{j-1} h_{k} y_{j k}
$$

Derived from the above we can see that the node meeting on the value of the node M-service is the cluster head, at the circumstances of the failure probability is $p$, the services of these cluster head are provided to meet the largest number of service requests.

If adopted the enumeration method to calculate (6), then the calculated time complexity is $O\left[\left(N^{\wedge} M\right) \times N\right]$, in the case of wireless sensor network with a huge number nodes, the cost of the calculated time can not be ignored. In order to simplify the complex calculations, the following heuristics should be applied to calculation.
Assuming the node which covers the maximum demand is unique, the heuristic begins with all cluster head located at this node, with, an indicator, set to 1.0 , and , a second indicator, set to 0.0. The heuristic then considers single node substitutions to this set of locations. At any stage in algorithm, we refer to the set of cluster head sites for which single node substitutions are being considered as the current location set. Initially the current location set has all cluster heads at the node which covers the maximum number of demands. Each set of current solution and replacing it with another location-performing a single node substitution-is termed a trial solution. In evaluating a trial solution, three results may occur:

(a) The trial solution is inferior to the current solution for all values of $p$ between $p^{* *}$ and $p^{*}$;

(b) The trial solution is better than the current solution for some value of $p$ in the interval $\left[p^{* *}, p^{*}\right]$;

(c) The trial solution is better than the current solution for $\mathrm{p}$ equal to $p^{*}$.

In case (a), the trial solution is discarded and a new trial solution is generated, if any remain to be generated. In case (b) the trial solution becomes the tentative solution, replacing the previous tentative solution if one existed. In addition, $p^{* *}$ is updated and set equal to the value of $p$ at which the trial solution becomes better than the current solution.

Again, a new trial solution is generated, if any remain to be generated. Note that, at any step of heuristic, $p^{* *}$ is the smallest value of $p$ at which the current solution is the best heuristic can find. For values of $p$ less than $p^{* *}$, the tentative solution is better than the current solution, $p^{* *}$ is set to 0.0 , and a new series of single node substitutions is initiated.

Once all possible single node substitutions that can be generated based on the current solution have been evaluated, if a tentative solution has been identified, it becomes the new current solution. In this case, $p^{*}$ is set equal to $p^{* *}$ (which will be smaller than $p^{*}$ ), $p^{* *}$ is then reset to 0.0 and a new series of single node substitutions is initiated. If a tentative solution does not exist, in which case $p^{* *}=0.0$, the algorithm stops.

So that we get the approximate location of the optimal $\left(S_{1}, S_{2}, S_{3}, \ldots, S_{m-2}, S_{m-1}, S_{m}\right)$. Its time complexity is $O(N \times M \times N)$. Heuristic algorithm processes as shown in Figure 1. This paper is about the use of the above heuristics algorithm to calculate the cluster head generation algorithm.

A variant of the maximum covering location problem that accounts for the possibility that facilities unable to respond to demands was formulated. The model 
is called the maximum expected covering location problem(MEXCLP). Several properties of the MEXCLP were proven. The most important of these is that for $0<p<1$, the solution to the MEXCLP will not include sominated nodes. A single node substitution heuristic algorithm was proposed. The algorithm finds good solutions for the full range of values of $p$. The heuristic also does not allow dominated nodes to enter and remain in any solution. Computational experience with the algorithm using a 55-node test problem was discussed. The heuristic indentified a know optimal solution to the maximum covering location problem(the $p=0$ case.)

The model assumes that the probability of a facility being busy is identical for all facilities. In addition the model assumes that the probability of a facility being busy is independent of whether or not the other facilities are busy. Both assumptions are clearly not strictly justified. The model may be reformulated as an integer nonlinear programming model that allows the probability of a facility being busy to depend on the facility site, thile retaining the independence assumption. Work on this model is currently underway and will be reported on in a later paper. Relaxing the independence assumption may be more difficult. However, a related study compared the expected coverage predicted by the MEXCLP model which assumes independence with that computed using Fitzsimmon's CALL model, which relaxes the independence assumption. The differences were not significant. Future research should attempt to relax the independent assumption and should examine its effect on location decisions as well as the expected coverage.

Several additional directions for future study may also be identified. First, an efficient optimal solution algorithm to the MEXCLP would clearly be desirable. Two approaches are currently under study: Bender's decomposition is being tested to solve a mixed integer formulation of the problem, and nonlinear programming approaches are being applied to the extension of the model discussed above that allows for locationspecific probabilities of a facility being busy. Second, additional computational experience with either heuristic or exact solutions to the model will enable us to strengthen the qualitative conclusions, Comparison of these results with those of simulation or queueing theory based models will also allow us to test the effects on both the objective function value and the location decisions of assuming that the probability of facilities being busy is the same for all facilities and that it is independent of tether or not other facilities

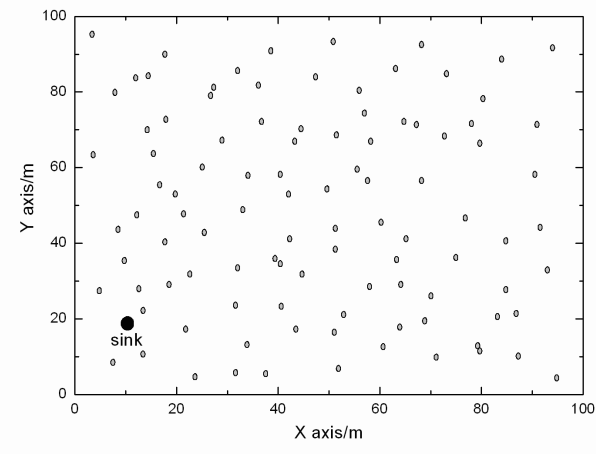

Figure 1 Randomly distribution sensor network nodes

are busy. Finally, optimization based models that relate these assumptions should also be developed.

\section{Simulation experiment results}

Here The MEXCLP algorithm assumptions the coverage radius of Dc is known, the coverage radius as the judge standards of the quality of service or service efficiency. The cluster head node coverage radius refers to the cluster head node of services only at a certain distance provided. The benefit of this is to ensure that within the scope cluster head provide accurate and efficient services for the sensor, from another angle to ensure the entire wireless sensor service capacity.

\section{A. Simulation 1:}

In the circumstance of not considering the case of cluster head node service failure, compared LEACH algorithm and this algorithm of cluster head distribution uniformity. Consider 100 nodes of the wireless sensor network, all the nodes in a random distribution of $100 m \times 100 m$ of the square area. Assume that all nodes have the same number of requests, and are equal to 1 . Nodes in the region distribution are shown in Figure 1. In case of not considering the probability of failure of service nodes in this wireless sensor networks, we select five cluster head nodes, using the traditional LEACH algorithm to generate the cluster head node distribution as shown in Figure 2.

It can be seen from Figure 2, in accordance with the LEACH algorithm the distribution of cluster head is uneven, which is decided by the characteristics of the algorithm, in LEACH algorithm each node produce a random number between 0 to 1 , if the number is less than Threshold $T(n)$, then release it is cluster head bulletin news, resulting in nodes generated randomness. 


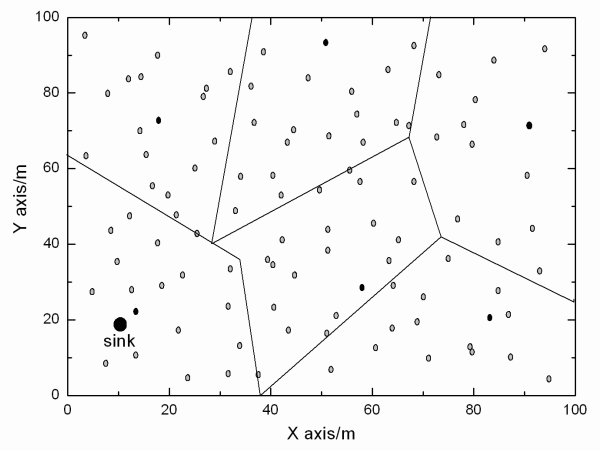

Figure 2 Clusters established by LEACH algorithm

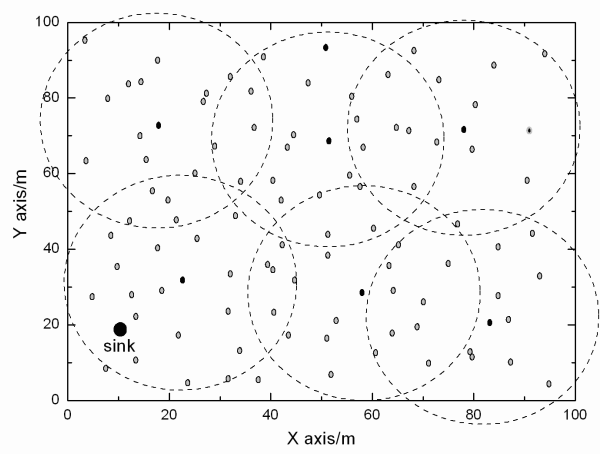

Figure 3 Clusters established by this algorithm

The cluster head generation algorithm puts forward by the paper is fully considered how to choose the cluster head node, made the request number most of the nodes which be serviced, the algorithm generated cluster head nodes as shown in Figure 3, from the map shows that this algorithm cluster head distribution more uniform than the LEACH algorithm.

From figure 2 and figure 3 , we can see that the LEACH algorithm has been widely used directly for it is easy to realize. Moreover the HEED algorithm modified based on the LEACH is also be used widely. Compared with these two algorithms, the clustering provided in this paper focus on the distribution and the service quality. So the uncertainty of communication and the demands covered by the cluster head was take into account carefully. In this view the provided algorithm is better than LEACH and HEED. The following is Comparing the capability between the LEACH algorithm and this algorithm.

\section{B. Simulation 2:}

In there we assumed that cluster head nodes provide data transmit service to other nodes probability for $\mathrm{p}$ circumstances (for all cluster head node, $p$ the same),

$$
p=\frac{\text { The number of forwarded datagrams }}{\text { the number of demanded forward datagram }}
$$

In this paper the NS-2 simulator was used. Researchers working in the area of wireless communications heavily rely on simulations to evaluate the performance of proposed algorithms. This is primarily because setting upon large scale wireless testbeds, and ensuring repeatability across multiple testbeds is a difficult task. The ns-2 simulator is one of the most widely used network level simulator for wireless research, since it is publicly available, and is open source.

The focus of this paper is the simulation of 802.11based wireless mesh networks in ns-2. As pointed out in, the current implementation of MAC and PHYlayer wireless models in ns- 2 does not abide by the following principles of wireless communication. The computation of SINR (Signal to Interference and Noise Ratio) should take into account the sum of the strengths of all the interfering signals instead of just the strongest interfering signal. The channel gain model should be comprehensive in that it should include distance based path loss, location dependent shadowing, and velocity dependent fading. The channel gains could be different in either direction due to shadow-fading, and the simulator should model such asymmetric link conditions. The simulator should also model fluctuations in the capacity of a link due to time variations resulting from fading.

Furthermore, ns-2 only allows for a common and fixed data rate for all the links in a mesh network. In real 802.11 hardware, depending on the channel conditions, a rate adaptation algorithm such as Auto Rate Fallback (ARF) chooses the highest possible data rate among the eight candidate data rates $(6,9,12,18,24$, 36, 48 and $54 \mathrm{Mbps}$ ) to suit the current channel conditions. Each of these data rates has different SINR requirements; the higher the SINR, the higher the sustainable data rate. A wireless link simulator should have support for such multi-rate and multi-SINR link model with link rate adaptation. Support for multirate links with ARF was contributed in, however this code relies on comparing the received signal strength to fixed thresholds for determining successful reception. Received power based decision is inaccurate since in real systems, the SINR determines the success/failure of a transmission. Using network simulation tool NS2 test algorithm capability, and compare itself to LEACH algorithm. Simulation Model composes of 100 nodes, 


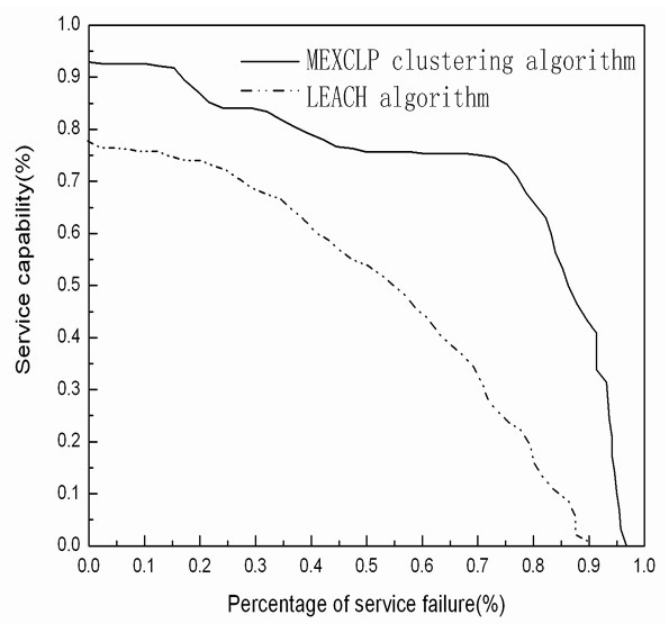

Figure 4 Algorithm capability curve

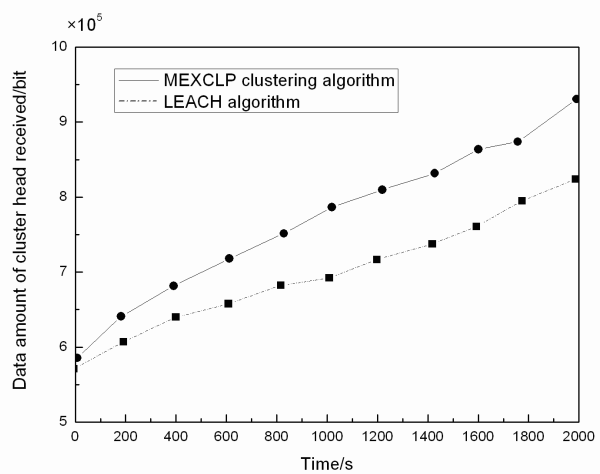

Figure 5 Sink node received data amount

randomly distributed within range $100 m \times 100 m$, and make sure 10 clusters head in networks. In any cluster any two member- nodes can communicate each other. The Sink node is located at $(10,20)$.

Figure 4 is about LEACH algorithm and MEXCLP algorithm capability curve. We can see that, with the cluster head of service failure rate increased, the service capability of LEACH algorithms and MEXCLP algorithm are reduced. But LEACH algorithm capability decline very significant with the service failure rate increase, in the case of service failure rate of 80 percent, the cluster head node is almost impossible to provide normal services. At the circumstance of MEXCLP failure rate of 78 percent, the cluster head node can still provide service; it is because the design of the algorithm gives full consideration to the possibility of failure service. Therefore, the cluster head node can provide better service at the circumstance of service failure rate still higher. Figure 5 shows the Sink node received data amounts changing with time. It can be seen that the data increase of Sink received with time changing, but used the MEXCLP algorithm Sink node receive the amount of data more than the amount of data used by LEACH algorithm. Therefore MEXCLP algorithm more effective than LEACH, and sent a greater amount of data.

\section{Conclusion}

Based on the MEXCLP principle a new clustering algorithm is put forward in this paper. Under the circumstance of provided failure rate, the nodes demands and the optimal location of the cluster heads are computed. The experiment results show that the distribution of the cluster heads is more uniform. With the same consumption between this algorithm put forward in this paper and the LEACH algorithm, the new algorithm can send more data. So it is easy to see that the new clustering algorithm is better than the traditional algorithm such as the LEACH.

\section{Acknowledgments}

This article is supported by Sponsored by College Innovation Group projects in Liaoning Province, China and Sponsored by Shenyang Science and Technology Plan projects, Shenyang, Liaoning, China.

\section{References}

[1] K Kurosawa, Y Desmedt. A New Paradigm of Hybrid Encryption SchemeC. BerlinSpringerVerlagAdvances in Cryptology CRYPTO 2004, 2004. 426-442.

[2] A. Agah, S. Das, K. Basu, and M. Asadi. Intrusion detection in sensor networks: A non-cooperative game approach. Proc. of ICWN'06. 2006, pp. 29-36.

[3] S. Capkun, L. Buttyan, and J. Hubaux. SECTOR: Secure tracking of node encounters in mult-hop wireless networks. Proc. of the ACM Workshop on Security of Ad Hoc and Sensor Networks. 2003, pp. 21-32.

[4] J. Carle and D. Simplot-Ryl. Energy-efficient area monitoring for sensor networks. Computer. Vol. 37, Issue 2, Feb., 2004, pp. 40-46.

[5] Zhang, R., Zhao, H., Labrador, M. A., A Grid-Based SinkLocation Service for Large-scale Wireless Sensor Networks, Int. conference on Communications and mobile computing, Vancouver, British Columbia, Canada, 689-694, July 2006.

[6] Akl, R., Sawant, U., Grid-based Coordinated Routing in Wireless Sensor Networks, IEEE Consumer Communications and Networking Conference (CCNC 2007), 5, . January 2007.

[7] G. Gupta, M. Younis, "Load-Balanced Clustering in Wireless Sensor Networks", Submitted to the IEEE 
International conference on communications (ICC 2003), Anchorage, Alaska, May 2003.

[8] K. Arisha, M. Youssef, M. Younis, "Energy-Aware TDMABased MAC for Sensor Networks," IEEE Workshop on Integrated Management of Power Aware Communications, Computing and Networking (IMPACCT 2002), May 2002.

[9] A. Cerpa and D. Estrin, "ASCENT: Adaptive SelfConfiguring Sensor Networks Topologies," Proc. INFOCOM 2002, New York, June 2002.

[10] Y. Sankarasubramaniam, O. B. Akan, and I. F. Akyildiz, "ESRT: event-to-sink reliable transport for wireless sensor networks," in Proc. ACM MOBIHOC, Annapolis, MD, Jun. 2003, pp. 177-188.

[11] Eduardo F. Nakamura, Heitor S. Ramos, Leandro A. Villas, Horacio A.B.F. de Oliveira, Andre L.L. de Aquino, Antonio A.F. Loureiro. A reactive role assignment for data routing in event-based wireless sensor networks, Computer Networks Volume 53, Issue 12, pp 1980-1996, August 2009.

[12] Awad, Abdalkarim and Sommer, Christoph and German,Reinhard and Dressler, Falko. [Virtual Cord Protocol (VCP): A Flexible DHT-like Routing Service for Sensor Networks]. 5th IEEE International Conference on Mobile Ad-hoc and Sensor Systems (IEEE MASS 2008), Atlanta, Georgia, USA, September 2008 .

[13] A.A. Abbasi and M. Younis. A survey on clustering algorithms for wireless sensor networks. Computer Communication,30(14-15):2826-2841, 2007.

[14] I. F. Akyildiz, T.Melodia, and K. R. Chowdhury. A survey on wireless multimedia sensor networks. Computer Networks, 51(4):921-960, 2007.

[15] I.F. Akyildiz, W. Su, Y. Sankarasubramaniam, and E. Cayirci. A survey on sensor networks. IEEE Communications Magazine, 2002.

[16] I. F. Akyildiz, W. Su, Y. Sankarasubramaniam, and E. Cayirci.Wireless sensor networks: a survey. Computer Networks, 38:393-422,2002.

[17] J. N. Al-karaki and A. E. Kamal. Routing techniques in wireless sensor networks: A survey. IEEE Wireless Communications, 11:6-28, 2004.

[18] P. Baronti, P.Pillai, V. W. C. Chook, S. Chessa, A. Gotta, and Y. F. Hu. Wireless sensor networks: A survey on the state of the art and the 802.15.4 and zigbee standards. Computer Communication, 30(7):1655$1695,2007$.

[19] W. R. Heinzelman, A. Chandrakasan, and H.Balakrishnan. Energyefficient

[20] communication protocol for wireless microsensor networks. In Proc. 33rd Hawaii International Conference on System Sciences, 2000.
[21] W.R. Heinzelman and P. Chandrakasan. An application-specific protocol

[22] architectures for wireless networks. IEEE Transactions on Wireless Communications, 1:660-670, 2002.

[23] V. Mhatre and C. Rosenberg. Homogeneous vs. heterogeneous clustered sensor networks: A comparative study. In Proceedings of 2004 IEEE International Conference on Communications ICC 2004, pages 3646-3651, 2004.

[24] L. Qing, Q. Zhu, and M. Wang. Design of a distributed energy-efficient clustering algorithm for heterogeneous wireless sensor networks. Computer Communication, 29:2230-2237,2006.

[25] T. J. Shepard. A channel access scheme for large dense packet radio networks. In Proc. ACM SIGCOMM, pages 219-230,1996.

[26] G. Smaragdakis, I. Matta, and A. Bestavros.SEP: A stable election protocol for clustered heterogeneous wireless sensor networks. In: Proc. of the Intl Workshop on SANPA, 2004. 